\title{
Faster walking under muscle fatigability: a strategy to improve stability or a warm-up effect?
}

\section{PAULO C. R. SANTOS ${ }^{1,2,3}$ | CLAUDINE LAMOTH4 | NICOLAS VUILLERME $^{5,6,7}$ | FABIO A. BARBIERI ${ }^{2,3}$}

1 Weizmann Institute of Science, Department of Computer Science and Applied Mathematics, Rehovot, Israel.

${ }^{2}$ São Paulo State University (UNESP), Human Movement Research Laboratory (MOVI-LAB), Department of Physical Education, Bauru, SP, Brazil.

3 São Paulo State University (UNESP), Graduate Program in Movement Sciences, Bauru, SP, Brazil.

4 University of Groningen, University Medical Center Groningen, Department of Human Movement Sciences, Groningen, The Netherlands.

5 University of Grenoble Alpes, AGEIS, Grenoble, France.

6 Institut Universitaire de France, Paris, France.

${ }^{7}$ LabCom Telecom4Health, University of Grenoble Alpes and Orange Labs, Grenoble, France.

\section{INTRODUCTION}

Conceptually, fatigability as a model is biologically relevant to explore gait-related adaptabilities since fatigability depletes the internal resources available ${ }^{1,2}$ and impacts peripherical and central mechanisms involved in muscle contraction ${ }^{1}$ and control of movements such as gait. ${ }^{3}$ Although the term "fatigue" is ubiquitous in sports and, more recently in healthy aging, the scope of fatigue usage and its effects on gait are still inconclusive. A potential reason for that is the widespread use of different fatigue terminologies which complicates translating fatigue to human performance. ${ }^{1} \mathrm{~A}$ taxonomy was proposed to accommodate the scope of fatigue for different contexts. The taxonomy conceptualizes fatigue as a trait level - fatigue experienced/reported during the preceding several days, normally ascribed to diseases or hospitalization - or a state - as a result of the continuous interaction between performance (a decline in an objective measure of performance) and perceived fatigability (changes in the self-reported fatigue sensations associated with the performance).

Regarding the fatigue state, several studies are experimentally inducing fatigability to examine gait-related adaptability. Normally, such studies design sustained physical or muscle effort protocols and measure fatigability by a decrease in the level of performance and/or increase in the perceived exertion. Therefore, it is reasonable to experimentally induce fatigability to safely examine how humans can adapt their functional tasks to this internal perturbation, ${ }^{3}$ mainly in populations in which fatigue state might be daily experienced.

Albeit the heterogeneity of endurance or muscle fatiguing protocols, similar adaptations in gait are observed.3,4 Among those, unexpected and counterintuitive 
increases in gait velocity ( 9\%) are consistently observed during overground walking after different fatiguing protocols in different populations (healthy young and older adults, Parkinson's disease) ${ }^{3-7}$ Besides, the mechanisms that explain such increases are still uncertain. Between the attempts to explain such increase, the literature uses two main theoretical arguments: a potential compensatory strategy to improve stability during walking; $;, 7$ and a response of the motor system in increasing the neural drive, ${ }^{8}$ similar to the effects of warm-up activities. ${ }^{9}$

Regarding the first argument (the compensatory strategy), the increase in gait velocity during overground walking after a fatiguing protocol was associated with a more stable gait pattern, ${ }^{4,7}$ because the faster walking velocity is accompanied by longer stride/step length and shorter step duration. ${ }^{4,5}$ Such gait adaptations are attributed to an improved balance control ${ }^{9}$ by seeking more stability in the anterior-posterior direction. For instance, the extrapolated center of mass, which is velocity-dependent, is anterior to the base of support, and the increased forward step (length) is a natural response to enhanced stability by decreasing the magnitude of the margin of stability during walking. ${ }^{9}$ Increasing the step length and decreasing the duration (to increase gait velocity) may be a safe, fast, effective compensatory strategy to increase the stability and avoid further consequences of fatigability on gait.

Considering the second argument (warm-up), fatiguing protocols might result in a preparatory elevation of muscle temperature, alertness, cardiovascular, and hormonal functions, invoking an increase in gait velocity. Since the metabolic and mechanical energy-wise minimal effort required during overground walking is low, gait performance would hardly be limited by fatiguing protocols. Additionally, typical fatigability-related increases in neural drive ${ }^{8}$ might also be an after-effect of warm-up activities that, in some tasks, result in increased muscle activation amplitude. ${ }^{9}$ Thus, the interpretation that fatigability causes compensatory increase neural drive to maintain the desired performance, may also be due to warm-up effects, mainly in submaximal tasks (as gait) in which the power/strength demands are below the levels of reductions in power/strength that fatiguing protocols could induce.

Although these two hypothetical explanations are described separately, those arguments might be complementary to elucidate the unexpected increase in gait velocity after fatigability. However, to the best of our knowledge, there is no direct evidence that could support these two hypothetical explanations. Accordingly, future studies aiming to verify the effects of performance fatigability on walking should measure fatigability in a broader domain (e.g., physiological and biomechanical) combined with assessments of neuromuscular control, kinetics, stability, and kinematics of gait. Fatiguing protocols should consider the specificity of muscles on gait and populations since most studies induced fatigability on knee extensors despite the fact that in old populations mainly, the plantarflexors thrust is the putative mechanism driving gait. Additionally, study designs should consider long-distance walking in a less controlled environment (outside walking) most studies assessed short-distance $(\sim 10 \mathrm{~m})$ lab walking tasks since it may interfere with movement economy and affect gait velocity. These protocol characteristics may avoid alternative explanations that the increase in gait velocity induced by performance fatigability may be a strategy of finishing the task rapidly. ${ }^{5}$ Also, protocols comparing maximum vs. self-selected speed after muscle fatigability would provide information on whether underlying neuromechanims related to fatigability-induced gait changes support 
the usual, yet unexpected, increase in gait velocity.

\section{REFERENCES}

1. Enoka RM, Duchateau J. Translating Fatigue to Human Performance. Med Sci Sport Exerc. 2016;48(11):2228-38.

2. Monjo F, Terrier R, Forestier N. Muscle fatigue as an investigative tool in motor control: A review with new insights on internal models and posture-movement coordination. Hum Mov Sci. 2015;44:225-33.

3. Santos PCR, Barbieri FA, Zijdewind I, Gobbi LTB, Lamoth C, Hortobágyi T. Effects of experimentally induced fatigue on healthy older adults' gait: A systematic review. PLOS One. 2019;14(12):e0226939. 10.1371/journal.pone.0226939

4. Barbieri FA, dos Santos PCR, Simieli L, Orcioli-Silva D, Van Dieën JH, Gobbi LTB. Interactions of age and leg muscle fatigue on unobstructed walking and obstacle crossing. Gait Posture. 2014;39(3):985-90. 10.1016/J.GAITPOST.2013.12.021

5. Granacher U, Wolf I, Wehrle A, Bridenbaugh S, Kressig RW. Effects of muscle fatigue on gait characteristics under single and dual-task conditions in young and older adults. $J$ Neuroeng Rehabil. 2010;7(1):56.

6. Morrison S, Colberg SR, Parson HK, Neumann S, Handel R, Vinik EJ, et al. WalkingInduced Fatigue Leads to Increased Falls Risk in Older Adults. J Am Med Dir Assoc. 2016;17(5):402-9. 10.1016/j.jamda.2015.12.013

7. Santos PCR, Gobbi LTB, Orcioli-Silva D, Simieli L, van Dieën JH, Barbieri FA. Effects of leg muscle fatigue on gait in patients with Parkinson's disease and controls with high and low levels of daily physical activity. Gait Posture. 2016;47:86-91.

10.1016/j.gaitpost.2016.04.002

8. Santos PCR, Lamoth CJC, Barbieri FA, Zijdewind I, Gobbi LTB, Hortobágyi T. Age-specific modulation of intermuscular beta coherence during gait before and after experimentally induced fatigue. Sci Rep. 2020;10(1). 10.1038/s41598-020-72839-1

9. Sotiropoulos K, Smilios I, Christou M, Barzouka K, Spaias A, Douda H, et al. Effects of warm-up on vertical jump performance and muscle electrical activity using half-squats at low and moderate intensity. J Sport Sci Med. 2010;9(2):326-31.

10. Hak L, Houdijk H, Steenbrink F, Mert A, Van der Wurff P, Beek PJ, et al. Speeding up or slowing down?: Gait adaptations to preserve gait stability in response to balance perturbations. Gait Posture. 2012;36(2):260-4. 10.1016/j.gaitpost.2012.03.005 
Citation: Santos PCR, Lamoth C, Vuillerme N, Barbieri FA. (2021). Faster walking under muscle fatigability: a strategy to improve stability or muscle post-activation effect?. Brazilian Journal of Motor Behavior, 15(3): 149-152.

Editors: Dr Fabio Augusto Barbieri - São Paulo State University (UNESP), Bauru, SP, Brazil; Dr José Angelo Barela São Paulo State University (UNESP), Rio Claro, SP, Brazil; Dr Natalia Madalena Rinaldi - Federal University of Espírito Santo (UFES), Vitória, ES, Brazil.

Section Editors (Current Opinion): Dr Luis Augusto Teixeira - University of São Paulo (USP), São Paulo, SP, Brazil; Dr Tibor Hortobágyi - University of Groningen, The Netherlands; Dr Renato de Moraes - University of São Paulo (USP), Ribeirão Preto, SP, Brazil.

Copyright:@ 2021 Santos, Lamoth, Vuillerme and Barbieri and BJMB. This is an open-access article distributed under the terms of the Creative Commons Attribution-Non Commercial-No Derivatives 4.0 International License which permits unrestricted use, distribution, and reproduction in any medium, provided the original author and source are credited.

Funding: This work was partially supported by CNPq (FAB, project number: \#445438/2014-2).

Competing interests: The authors have declared that no competing interests exist.

DOI: https://doi.org/10.20338/bjmb.v15i3.241 